\title{
Comparative Transcriptome Analysis of Rice Cultivars Resistant and Susceptible to Rhizoctonia Solani AG1-IA
}

\section{Yan Wang}

Shenyang Agricultural University

Hang Luo

Shenyang Agricultural University

Haining Wang

Shenyang Agricultural University

Zongjing Xiang

Shenyang Agricultural University

Songhong Wei ( $\nabla$ songhongw125@163.com )

Shenyang Agricultural University

Wenjing Zheng

Liaoning Rice Research Institute

\section{Research Article}

Keywords: Rice sheath blight, Transcriptome, Resistance mechnism, Phenylalanine metabolism

Posted Date: December 10th, 2021

DOI: https://doi.org/10.21203/rs.3.rs-1112960/v1

License: (1) This work is licensed under a Creative Commons Attribution 4.0 International License.

Read Full License 


\section{Abstract}

Background: Rice sheath blight, which is caused by Rhizoctonia solani, is the most destructive disease affecting rice production, but the resistance mechanism to this pathogen has not been fully elucidated.

Results: In this study, we selected two rice cultivars based on their resistance to the pathogen and analyzed and compared the transcriptomic profiles of two cultivars, the moderately resistant variety Gangyuan8 and the highly susceptible variety Yanfeng47, at different time points after inoculation. The comparative transcriptome profiling showed that the expression of related genes gradually increased after pathogen inoculation. The number of differentially expressed genes (DEGs) in Yanfeng47 was higher than that in Gangyuan8, and this result revealed that Yanfeng 47 was more susceptible to fungal attack. At the early stage ( 24 and $48 \mathrm{~h}$ ), the accumulation of resistance genes and a resistance metabolism occurred earlier in Ganguan8 than in Yanfeng47, and the resistance enrichment entries were more abundant in Ganguan8 than in Yanfeng47.

Conclusions: Based on the GO and KEGG enrichment analyses at five infection stages, we concluded that phenylalanine metabolism and the jasmonic acid pathway play a crucial role in the resistance of rice to sheath blight. Through a comparative transcriptome analysis, we preliminarily analyzed the molecular mechanism responsible for resistance to sheath blight in rice, and the results lay the foundation for the development of gene mining and functional research on rice resistance to sheath blight.

\section{Background}

Rice sheath blight (RHB) is one of the three major rice diseases that seriously affects the yield and quality of rice and was first discovered by Japanese researchers in 1910 [10,24, 29]. The planting of resistant varieties is considered the most effective method for controlling the disease [36]. To date, varieties with high resistance or immunity that carry major genes have not been identified [39]. To date, many QTLs for rice resistance to sheath blight have been reported, and pyramiding multiple QTLs has proven to be a feasible strategy for improving rice resistance to disease $[5,6,19,22,40]$. Rhizoctonia solani, a soil-borne Basidiomycete fungus, can be divided into 14 groups, and among these groups, AG1 is the main hyphal fusion group and the major pathogenic bacterium responsible for rice sheath blight [27, 38].

With the development of sequencing technology, next-generation sequencing technology is characterized by high throughput and low cost [13] and has opened up new research directions involving the study of gene structure, expression and function and greatly promoted omics analyses [32]. The physiological and biochemical reactions and phenotypic changes induced by plants in response to pathogen infection are determined by related genes [8]. These genes participate in multiple molecular regulation pathways and cross each other to form a set of complex disease resistance regulation networks to ultimately achieve disease resistance $[20,31]$. Due to their complexity, rice disease resistance mechanisms are difficult to analyze using traditional methods. The defense mode, response and pathogenic mechanism during the interaction between rice and fungi are very complex [37]. Although many studies have been performed, no 
breakthroughs have been achieved, and research on rice sheath blight still faces many difficulties [28]. In this study, RNA-seq technology was used to conduct a comparative transcriptome analysis of infected leaves at the tillering stage. Gangyuan8 (GG), a moderately resistant variety, and Yanfeng47 (YY), a susceptible variety, were analyzed at 0, 24, 48, 72 and $96 \mathrm{~h}$ after AG1 IA inoculation. A transcriptome analysis was performed to explore the resistance mechanism to rice sheath blight. Bioinformatics and qRT-PCR analyses were performed to investigate how two rice cultivars induce resistance or susceptibility to $R$. solani and to identify the metabolic pathways associated with this response. To our knowledge, this study involves the first use of production varieties for a comparative transcriptome profiling analysis of the response to AG1 IA infection.

\section{Results}

\section{Selection and determination of sampling time points}

To determine the time points for transcriptome sampling, we used fugus cake cultured with $R$. solani AG1 IA for 2 days, inoculated the middle of the leaves of the different varieties at the tillering stage and wrapped the leaves with plastic wrap. The results showed that $24 \mathrm{~h}$ after inoculation, mycelia were attached to the leaf surface of Yanfeng47. In addition, $48 \mathrm{~h}$ after inoculation, disease spots began to form on the leaf surface of Yanfeng47, and Gangyuan8 also started to show disease spots. Seventy-two hours after inoculation, the lesion area of Yanfeng47 had expanded, and Gangyuan8 also showed obvious lesion. Ninety-six hours after inoculation, the leaves of Yanfeng47 had wilted, and the lesion area of Gangyuan8 was larger. Consistent with the results of previous studies, the resistance of Gangyuan8 and Yanfeng47 to $R$. solani AG1 IA showed significant differences (Supplementary Figure S1).

\section{RNA-seq results}

To investigate the changes in the gene expression profiles of Gangyuan8 and Yanfeng47 leaves during the initial stage of AG1 infection, we performed a transcription analysis of leaves after AG1 IA inoculation by high-throughput sequencing. Transcriptome data were generated from the leaves at different time points. A transcriptome analysis of each variety was performed using 4 time points and 3 biological replicates of each time point. Thirty samples yielded $236.04 \mathrm{~Gb}$ of clean data, and $6.18 \mathrm{~Gb}$ of clean data were obtained from each sample. The Q30 base percentage was at least $93.91 \%$. The clean reads from each sample were sequenced using the designated reference genome, and the alignment efficiency ranged from $76.72-95.72 \%$. To examine the quality of the biological replicates, we calculated Pearson correlation coefficient (PCC) values for each pair of samples and performed a cluster analysis, which showed that the 96-h samples were clustered far from their replicated samples (Supplementary Figure S2). We also found that the 48- and 72-h samples were clustered together within the same species, which might indicate that the changes in gene expression within the samples from 48 to $72 \mathrm{~h}$ were not obvious. This result suggested that the clustering order of all the samples was roughly consistent with the sampling time. These samples were also subjected to a principal component analysis (PCA) (Supplementary Figure S3). The first and second principal components (PC1 and PC2) showed that the 
96-h samples were separated from the other samples. The 0- and 24-h Yanfeng47 samples were also separated by a long distance, which indicated that the susceptible varieties were more responsive to stress, and this this finding was consistent with the results shown in Figure S1.

\section{Differentially expressed genes}

Differentially expressed genes (DEGs) were identified by comparing the gene expression data obtained from Gangyuan8 and Yanfeng47 leaves at five time points. At all tested time points after inoculation, the number of upregulated genes exceeded the number of downregulated genes in both cultivars, and the number of upregulated genes in Yanfeng47 leaves was higher than that in Gangyuan8 leaves (Table 1). In both cultivars, the number of DEGs continued to increase from 0 to $96 \mathrm{~h}$ after inoculation. In Gangyuan8, the number of DEGs identified $96 \mathrm{~h}$ after inoculation was significantly higher than that found at other time points, and the number of DEGs showed an increasing trend from 0 to $96 \mathrm{~h}$ after inoculation. The results showed that the influence of the pathogen on the Gangyuan8 leaves decreased from 0 to $96 \mathrm{~h}$ after inoculation through a self-regulation mechanism. This study compared the DEGs of the two varieties at the same time points (GG24-YY24, GG48-YY48, GG72-YY72 and GG96-YY96; Figure 1) and compared the DEGs of each variety at the different time points (GG24 h, GG48 h, GG72 h, GG96 h, YY24 h, YY48 h, YY72 $h$ and YY96 h; Figure 2). As shown in Figure 3, 1656 and 3688 DEGs were identified in the two varieties at 72 and $96 \mathrm{~h}$ after inoculation, respectively, and the number of these DEGs was higher than that at other time points. In Gangyuan8 leaves, 555 DEGs were shared among the DEGs at 24, 48, 72 and $96 \mathrm{~h}$ after inoculation, and these included 409 upregulated genes and 122 downregulated genes. A total of 2460 DEGs were found in the Yanfeng47 leaves, and these included 1135 upregulated genes and 1159 downregulated genes. As indicated in Figure 4, from 24 to $96 \mathrm{~h}$ after inoculation, the number of DEGs in Yanfeng47 leaves was higher than that in Gangyuan8 leaves.

Table 1

Statistics on the number of differentially expressed genes (DEGs)

\begin{tabular}{|clll|}
\hline DEG Set & Number of DEGs & Upregulated & Downregulated \\
\hline GG24 h & 1560 & 1186 & 374 \\
GG48 h & 2780 & 2180 & 600 \\
\hline GG72 h & 3171 & 2406 & 765 \\
\hline GG96 h & 5953 & 3269 & 2684 \\
\hline YY24 h & 5783 & 3419 & 2364 \\
\hline YY48 h & 5804 & 3483 & 2321 \\
\hline YY72 h & 6216 & 3994 & 2222 \\
\hline YY96 h & 7112 & 4020 & 3092 \\
\hline
\end{tabular}

\section{Functional enrichment analysis}


The enriched DEGs between the control and inoculation treatments were classified by GO annotation, and we found that the enriched DEGs were involved in multiple biological activities (Figure S4). All unigenes were annotated in the $\mathrm{GO}$ database and classified into three main categories. Among biological processes, "chloroplast envelope", "chloroplast stroma" and "plasma membrane" were the three processes showing the highest degree of gene enrichment. The analysis of molecular functions revealed that "iron ion binding" was the only process with the highest degree of enrichment. In the cellular component category, "pentose-phosphate shunt", "response to cadmium ion", "response to cold", and "response to salt stress" were the four processes with the highest enrichment degree.

In this study, some GO pathways were enriched in only a few DEGs (Figure S5). GO: 0016168, GO: 0018298, GO: 0016023 and GO: 0009523 were enriched only in the GG_0_VS_24 and GG_0_VS_48 $\mathrm{h}$ comparisons. This result indicated that the functional pathways involving these genes may be related to the early response of the varieties to pathogens. Our results suggest that at early time points in the riceAG1 IA interaction, the genes related to chlorophyll binding, protein-chromophore linkage, cytoplasmic membrane-bounded vesicle and photosystem II are more active in Gangyuan8 leaves than in Yanfeng47 leaves.

\section{Analysis of the gene expression profiles of rice cultivars}

We also wanted to further analyze whether a certain gene is only expressed in the early stage of GG but not in YY; thus, these genes are likely related to the difference in the responses of the varieties to AG1 IA. Therefore, we extracted all DEGs and used them to generate expression profiles (Figure 3 ). In the diagram, the red colors represent high expression, and the blue colors represent low expression. The GG sample is shown on the left, and the YY sample is presented on the right. A total of 11 clusters were obtained, and the expression information of each cluster is shown in the line chart on the right of Figure 3. In this line chart, the red color represents the GG sample, and the black color represents the YY sample, as in cluster 9. The expression of these genes was high at $24 \mathrm{~h}$ and then decreased, and this profile is probably related to the early response of rice varieties to the pathogen. At the same time, the expression level of cluster 9 in the GG sample was higher than that in the YY sample, which may be due to the high expression of genes involved in resistance to infection in GG; thus, GG was identified as a disease-resistant variety. A similar trend was found for cluster 10 . The analysis of clusters 9 and 10 obtained for GG revealed that the expression level of the genes related to cluster 9 increased continuously before $24 \mathrm{~h}$ and then decreased continuously after $24 \mathrm{~h}$. In cluster 10, the increase and decreased occurred at 24 and $72 \mathrm{~h}$, respectively. The GO enrichment results showed that the related genes in cluster 10 were mainly concentrated in the integrity of the membrane, metabolic process and cytoplasmic membrane-bound vesicle (Figure S6). The expression of cluster 11 in GG and YY remained highly after inoculation. In addition, the genes in cluster 11 were enriched in the response to salt stress, heme binding and extracellular matrix.

\section{Metabolic pathway analysis after AG1 IA inoculation}


We selected the DEGs of the two varieties at different time points for KEGG enrichment analysis (Figure S7). We found that some of the metabolic enrichments associated with resistance, namely, phenylpropanoid biosynthesis, alpha-linolenic acid metabolism, phagosomes, and the TCA cycle, were only enriched in Gangyuan8, and plant-pathogen interactions were only enriched in Yanfeng47. Based on the results of the KEGG enrichment analysis of the DEGs in Gangyuan8 at different stages, we found that immune-related metabolism was significantly enriched the initial stage of infection, and phenylpropanoid biosynthesis was significantly enriched after $24 \mathrm{~h}$ and was only enriched in the resistant variety. Alpha-linolenic acid metabolism, which is highly related to jasmonic acid metabolism, was significantly enriched at $48 \mathrm{~h}$. At the same time point, other resistance-related metabolisms, such as diterpenoid biosynthesis, were also significantly enriched. Based on the previous expression profile analysis, we preliminarily confirmed that the genes in clusters 9, 10 and 11 were involved in the disease resistance pathway of rice. To identify potential regulatory genes closely related to phenylalanine metabolic pathways, we identified DEGs by comparing the expression changes between the two varieties. At $96 \mathrm{~h}$, the expression level of genes related to phenylalanine metabolism in the GG samples was significantly higher than that in the YY samples, and the difference between the two varieties can be seen in the heatmap (Figure 4). Jasmonic acid is an important signaling molecule in plants, and the biosynthesis of jasmonic acid in plants originates from linolenic acid. To explore the role of the jasmonic acid pathway in rice sheath blight resistance, we drew a heatmap of the related genes involved in alphalinolenic acid metabolism and compared the differences between the two varieties. The results showed that the expression of genes related to alpha-linolenic acid metabolism was higher in the GG samples than in the YY samples. As shown in Figure 5, the expression level of related genes in the GG samples was always significantly higher than that in the YY samples. The expression levels of genes closely related to plant hormone signal transduction in the leaves of the two cultivars are shown in Figure 6. From 24 to $72 \mathrm{~h}$, the expression levels of related genes in the GG samples were significantly higher than those in the YY samples.

\section{Quantitative RT-PCR (qRT-PCR) validation of DEGs}

To verify the reliability of the sequencing results, we selected 12 genes that were expressed in both the GG and YY samples for qPCR verification. The results showed that the expression trends of the 12 genes were similar to the sequencing results, which indicated that the sequencing results used in this study were relatively reliable (Figure S8).

\section{Discussion}

A large number of pathogenic microorganisms have been found in the growing environment of rice and other plants and therefore threaten the normal life activities of plants [15]. To cope with this challenge, rice and other plants have developed a complex and elaborate innate immune system during their longterm coevolution with pathogens and utilize this system to recognize and resist various pathogenic microorganisms, such as fungi, bacteria, viruses, and nematodes, and to further trigger the immune defense response $[18,30]$. In this study, we used RNA-seq technology to sequence the transcriptome of 
Gangyuan8 and Yanfeng47 leaves at different time points after AG1 IA infection and explored the resistance mechanism of rice to sheath blight by comparing the gene expression changes of materials with different resistance levels. The results showed that the two cultivars exhibited two different gene regulation patterns in response to AG1 IA infection. The results of the transcriptome analysis revealed that the related DEGs increased over time after infection. At different time points, the number of genes in Yanfeng47 was higher than that in Gangyuan8, which indicated that Yanfeng47 was under greater infection pressure.

Plant hormones such as salicylic acid, jasmonic acid and ethylene, which are important signaling molecules in the immune response of rice, have been found to extensively mediate the disease resistance response of rice [23] and help plants adapt to stress by mediating the expression of downstream genes [25]. A number of studies have shown that plant hormones are important secondary transduction signals in the rice defense response and are crucial for the activation of genes related to rice disease resistance and the transmission of downstream defense signals $[1,7,21,33]$. Among these signals, salicylic acid and jasmonic acid are the two most important defense response hormones $[2,9,17]$. Through a series of precise positive and negative regulatory effects, these hormones can achieve a balance between growth and the stress response in rice. Jasmonic acid is a cyclopentanone derived from linolenic acid that plays a regulatory role in plant development and fungal infection [11]. In this study, genes involved in alphalinolenic acid metabolism in Gangyuan8 leaves were significantly enriched at $24 \mathrm{~h}$, which indicated that the jasmonic acid signaling pathway plays a very important role in the infection of Gangyuan8 leaves with AG1 IA. It has been speculated that jasmonic acid is involved in the immune response and related defense response of rice at the early stage of infection.

Plant secondary metabolites play an important role in plant life and are widely involved in plant growth and development, signal transduction and pathogen defense [35]. The phenylpropanoid pathway is also involved in the biosynthesis of many plant defensive compounds, including flavonoids, lignin, condensed tannins, hydroxycinnamic acid, coumarins and stilbenes [4, 26]. Arabidopsis, soybean and pepper have multiple PAL genes $[3,12]$. PALs are responsive to pathogen infection and may positively regulate the accumulation of salicylic acid in maize to resist sugarcane mosaic virus (SCMV) infection [16, 34]. The KEGG results showed that phenylalanine metabolism plays an important role in the resistance of Gangyuan8 to Rhizobacteria solani infection, particularly at the early stage of infection. An analysis of the related genes in clusters 9, 10 and 11 showed that phenylalanine ammonia lyase genes were activated in leaves after AG1 IA inoculation. In this study, two differentially expressed PAL genes that participate in phenylalanine metabolic pathways were identified. For example, Os04g0518400 and Os12g0520200 are thought to be involved in phenylalanine biosynthesis.

\section{Conclusions}

In summary, the comparative transcriptome analysis and gene expression pattern comparison of Gangyuan8 and Yanfeng47 infected with Rhizoctonia solani comprehensively identified the defense mechanism involved in the response of rice to AG1 IA infection. Multiple resistance pathways were 
activated by AG1 IA infection, and different genes participated in the defense response and signal transduction. This result suggested that the response of rice to pathogens is regulated by multiple gene networks. Based on the comparative transcriptome analysis, a number of metabolic pathways associated with resistance were found to be significantly activated, and we focused on the biosynthesis of jasmonic acid and phenylalanine metabolism. Resistance genes belonging to the PAL gene family were identified in this study, and the upregulated expression of PAL genes indicated that these genes may play an important role in the resistance mechanism of rice to AG1 IA. At the early stage of infection, PAL genes were activated in the resistant varieties, which indicated that the initial defense mechanism of rice against AG1 IA may mainly involve self-prevention. A transcriptome analysis helps us better explore the molecular mechanism of rice sheath blight resistance, and the data obtained in this study can also be used to screen for candidate resistance genes that can be used for the genetic improvement of germplasm resources.

\section{Methods}

\section{Plant growth and infection assay}

All accession seeds used in this study were provided by Shenyang Agriculture University. The experiments were performed in Shenyang. The plots of the cultivars consisted of four-meter-long rows of 20 plants with $30-\mathrm{cm}$ spacing between the rows. No fungicide was used throughout the growth period. The seeds were sown in mid-April, and the seedlings were transplanted at the end of May. Rice plants at the tillering stages were inoculated in the experiment. The methods and disease assessment scale of rice sheath blight were based on the Technical Specifications for the Identification of Resistance to Rice Sheath Blight (NY/T 2720 - 2015). R. solani strain Y-36, which was initially collected in Liaoning Province in northern China, was obtained from Shenyang Agriculture University and cultured on potato dextrose agar (PDA) plugs [14]. The cells were cultured for 7 days until the mycelia grew throughout the plate. When the plants were at the late tillering stage, the fugus cakes were attached to the upper 3 leaf sheaths of rice plants and wrapped with sealing film, which is beneficial for moisturizing. Three centimeters of the leaves were cut at five times points after inoculation $(0,24,48,72$ and $96 \mathrm{~h})$. Three biological replicates and random leaf samples were collected. The samples were rapidly frozen in liquid nitrogen and stored at $-80^{\circ} \mathrm{C}$. All the samples were sent to Beijing Biomarker Technologies for transcriptome sequencing.

\section{RNA-seq data processing}

The preprocessed RNA sequence readings were mapped to each reference genome using Cufflinks, TopHat2, and Bowtie software. The reference genome was MSU7. The Cufflinks component was used for the annotations and for the isolation of new genes from known genes for downstream analysis. The readings from each sample were then mapped to the rice genome using TopCat2.1.1. The untagged sequential reads were aligned with these composite sequences using Bowtie.

\section{Screening of DEGs}


The DESeq R algorithm was used for the screening of DEGs. To improve the detection rate of differentially expressed transcripts, the data were filtered to remove the transcripts with the lowest total number of $40 \%$. The estimated size factor function was used to estimate the effective library size for the normalization of transcript counting. The dispersion was estimated using the estimated dispersion function. The nbinomTest function was used to determine whether there was differential expression between the two conditions. The false discovery rate (FDR) was controlled at $5 \%$ using the BenjaminiHochberg method. Transcript per million (TPM) values were used to measure the proportion of transcripts in the RNA library. The overlap of differentially expressed transcripts identified from the comparison was analyzed using a Venn diagram.

\section{Validation of DEGs by quantitative real-time PCR (qRT-PCR)}

To verify the reliability of the sequencing results of DEGs, 12 DEGs identified from Gangyuan8 and Yanfeng47 were detected by qPCR, and the specific primers are shown in Table S1. The experimental protocol was based on the Vazyme AceQ qPCR SYBR Green Master Mix experimental procedure.

\section{Pattern mining based on time points and gene expression analysis}

To analyze the control and treatment groups at different time points, we designed a series of formulas, including the conditional factor, the time factor and their interaction. Under this premise, we used a simplified model without interaction terms to conduct a likelihood ratio test to test whether this condition induced changes in gene transcription at any time point after the control time point $(0 \mathrm{~h}$ after inoculation). A total of 7472 DEGs were identified between Gangyuan8 and Yanfeng47 leaves. We conducted a coexpression analysis of all tissues using the R package "pheatmap" (https://cran.rproject.org/web/packages/pheatmap/index.html). Before running the software, we calculated the relative expression values of the genes by dividing their expression level by their maximum observed FPKM value. The relative expression levels were used as input for "pheatmap" and clustered using the hierarchical clustering method.

\section{Functional enrichment analysis}

EggNOG-mapper software (v2) was applied to annotate genes in rice (http://eggnog-mapper.embl.de/) using the protein sequences as input files. The GO and KEGG enrichment analyses of DEGs was implemented using the "clusterProfiler" R package. GO and KEGG terms with FDR $<=0.05$ were defined as enriched.

\section{Abbreviations}

RHB: Rice sheath blight; GG: Gangyuan8; YY: Yanfeng47; R. Solani: Rhizoctonia solani; PDA: Potato dextrose agar; DEG: Differentially expressed genes; FDR: False discovery rate; TPM: Transcript per million; PCC: Pearson correlation coefficient; PCA: Principal component analysis. 


\section{Declarations}

\section{Availability of data and materials}

RNA-seq reads were deposited in NCBI database under project accession PRJNA782381. All data generated or analyzed during this study are included in this article and its Additional fles. The datasets generated and analyzed during the current study are available from the corresponding author on reasonable request.

\section{Acknowledgements}

We thank Prof. Wenjing Zheng at Liaoning Rice Research Institute for the rice varieties. AJE (https://www.aje.com/) for its linguistic assistance during the preparation of this manuscript.

\section{Funding}

This work was supported by the the earmarked fund for China Agriculture Research System (CARS-01).

\section{Author details}

${ }^{1}$ College of Plant Protection, Department of Plant Pathology, Shenyang Agricultural University, Shenyang 110866, Liaoning, China

2 Liaoning Rice Research Institute, Shenyang 110101, Liaoning, China

\section{Authors's contributions}

Yan Wang: Writing-Original Draft, Data curation. Hang Luo: Data curation. Haining Wang: Validation. Zongjing Xiang: Investigation. Songhong Wei: Writing - Review \& Editing. Wenjing Zheng: Resources.

\section{Ethics declarations}

\section{Ethics approval and consent to participate}

The seed materials of the cultivars used in this study were commercially available. Plant materials used in the analysis are maintained in accordance with the institutional guidelines of Shenyang Agricultural University, China. This article did not contain any studies with human participants or animals and did not involve any endangered or protected species.

\section{Consent for publication}

The authors consent to the above manuscript being published in BMC Genomics.

\section{Competing interests}

The authors declare that they have no competing interests. 


\section{References}

1. Alazem, M.; Lin, N.S., Roles of plant hormones in the regulation of host-virus interactions. $M O L$ PLANT PATHOL 2015, 16, (5), 529-40.

2. An, C.; Mou, Z., Salicylic acid and its function in plant immunity. J INTEGR PLANT BIOL 2011, 53, (6), 412-28.

3. Bednarek, P.; Osbourn, A., Plant-microbe interactions: chemical diversity in plant defense. SCIENCE 2009, 324, (5928), 746-8.

4. Chakraborty, A.K.; Dustin, M.L.; Shaw, A.S., In silico models for cellular and molecular immunology: successes, promises and challenges. NAT IMMUNOL 2003, 4, (10), 933-6.

5. Channamallikarjuna, V., Sonah, H., Prasad, M., Rao, G. J. N., Chand, S., Upreti, H. C., Singh, N. K., Sharma, T. R., Identification of major quantitative trait loci qSBR11-1 for sheath blight resistance in rice. MOL BREEDING 2010, 25, (1).

6. Chen, Z.X.; Zhang, Y.F.; Feng, F.; Feng, M.H.; Jiang, W.; Ma, Y.Y.; Pan, C.H.; Hua, H.L.; Li, G.S.; Pan, X.B.; Zuo, S.M., Improvement of japonica rice resistance to sheath blight by pyramiding qSB-9(TQ) and qSB-7(TQ). FIELD CROP RES 2014, 161, 118-127.

7. De Vleesschauwer, D.; Xu, J.; Hofte, M., Making sense of hormone-mediated defense networking: from rice to Arabidopsis. FRONT PLANT SCI 2014, 5, 611.

8. Eizenga, G.C.; Prasad, B.; Jackson, A.K.; Jia, M.H., Identification of rice sheath blight and blast quantitative trait loci in two different O-sativa/O-nivara advanced backcross populations. $M O L$ BREEDING 2013, 31, (4), 889-907.

9. Gan, L.; Wu, H.; Wu, D.; Zhang, Z.; Guo, Z.; Yang, N.; Xia, K.; Zhou, X.; Oh, K.; Matsuoka, M.; Ng, D.; Zhu, C., Methyl jasmonate inhibits lamina joint inclination by repressing brassinosteroid biosynthesis and signaling in rice. PLANT SC/2015, 241, 238-45.

10. Helliwell, E.E.; Wang, Q.; Yang, Y., Transgenic rice with inducible ethylene production exhibits broadspectrum disease resistance to the fungal pathogens Magnaporthe oryzae and Rhizoctonia solani. PLANT BIOTECHNOL J 2013, 11, (1), 33-42.

11. He, Y.; Zhang, H.; Sun, Z.; Li, J.; Hong, G.; Zhu, Q.; Zhou, X.; MacFarlane, S.; Yan, F.; Chen, J., Jasmonic acid-mediated defense suppresses brassinosteroid-mediated susceptibility to Rice black streaked dwarf virus infection in rice. NEW PHYTOL 2017, 214, (1), 388-399.

12. Huang, J.; Gu, M.; Lai, Z.; Fan, B.; Shi, K.; Zhou, Y.H.; Yu, J.Q.; Chen, Z., Functional analysis of the Arabidopsis PAL gene family in plant growth, development, and response to environmental stress. PLANT PHYSIOL 2010, 153, (4), 1526-38.

13. Jain, M., Next-generation sequencing technologies for gene expression profiling in plants. BRIEF FUNCT GENOMICS 2012, 11, (1SI), 63-70. 
14. Jia, Y.; Correa-Victoria, F.; McClung, A.; Zhu, L.; Liu, G.; Wamishe, Y.; Xie, J.; Marchetti, M.A.; Pinson, S.R.M.; Rutger, J.N.; Correll, J.C., Rapid Determination of Rice Cultivar Responses to the Sheath Blight Pathogen Rhizoctonia solani Using a Micro-Chamber Screening Method. PLANT DIS 2007, 91, (5), 485-489.

15. Jones, J.D.G.; Dangl, J.L., The plant immune system. NATURE 2006, 444, (7117).

16. Kim, D.S.; Hwang, B.K., An important role of the pepper phenylalanine ammonia-lyase gene (PAL1) in salicylic acid-dependent signalling of the defence response to microbial pathogens. J EXP BOT 2014, 65, (9), 2295-306.

17. Marjamaa, K.; Kukkola, E.M.; Fagerstedt, K.V., The role of xylem class III peroxidases in lignification. $J$ EXP BOT 2009, 60, (2), 367-376.

18. Molla, K.A.; Karmakar, S.; Molla, J.; Bajaj, P.; Varshney, R.K.; Datta, S.K.; Datta, K., Understanding sheath blight resistance in rice: the road behind and the road ahead. PLANT BIOTECHNOL J 2020, 18, (4), 895-915.

19. Noor, A.; Meier, A.; Matthew, G.; Gongora, Y.S.; Oard, J.; Jaiswal, P., Loss of premature stop codon in the Wall-Associated Kinase 91 (OsWAK91) gene confers sheath blight disease resistance in rice. bioRxiv 2019.

20. Okubara, P.A.; Dickman, M.B.; Blechl, A.E., Molecular and genetic aspects of controlling the soilborne necrotrophic pathogens Rhizoctonia and Pythium. PLANT SCI 2014, 228, (SI), 61-70.

21. Pieterse, C.M.; Leon-Reyes, A.; Van der Ent, S.; Van Wees, S.C., Networking by small-molecule hormones in plant immunity. NAT CHEM BIOL 2009, 5, (5), 308-16.

22. Pieterse, C.M.; Van der Does, D.; Zamioudis, C.; Leon-Reyes, A.; Van Wees, S.C., Hormonal modulation of plant immunity. Annu Rev Cell Dev Biol 2012, 28, 489-521.

23. Robert-Seilaniantz, A.; Grant, M.; Jones, J.D., Hormone crosstalk in plant disease and defense: more than just jasmonate-salicylate antagonism. ANNU REV PHYTOPATHOL 2011, 49, 317-43.

24. Sandhya, R.K.; Anuprita, R.; Santosh, K.S.; Krishnendu, C.; Ramani, K.S., Physiological Basis of Stagnant Flooding Tolerance in Rice. RICE SCI2017, 24, (02), 73-84.

25. Shigenaga, A.M.; Argueso, C.T., No hormone to rule them all: Interactions of plant hormones during the responses of plants to pathogens. SEMIN CELL DEV BIOL 2016, 56, 174-189.

26. Shine, M.B.; Yang, J.; El-Habbak, M.; Nagyabhyru, P.; Fu, D.; Navarre, D.; Ghabrial, S.; Kachroo, P.; Kachroo, A., Cooperative functioning between phenylalanine ammonia lyase and isochorismate synthase activities contributes to salicylic acid biosynthesis in soybean. NEW PHYTOL 2016, 212, (3), 627-636.

27. Shu, C.; Zhao, M.; Anderson, J.P.; Garg, G.; Singh, K.B.; Zheng, W.; Wang, C.; Yang, M.; Zhou, E., Transcriptome analysis reveals molecular mechanisms of sclerotial development in the rice sheath blight pathogen Rhizoctonia solani AG1-IA. FUNCT INTEGR GENOMIC 2019, 19, (5), 743-758.

28. Silva, J.; Scheffler, B.; Sanabria, Y.; De Guzman, C.; Galam, D.; Farmer, A.; Woodward, J.; May, G.; Oard, J., Identification of candidate genes in rice for resistance to sheath blight disease by whole genome sequencing. THEOR APPL GENET 2012, 124, (1), 63-74. 
29. Singh, P.; Mazumdar, P.; Harikrishna, J.A.; Babu, S., Sheath blight of rice: a review and identification of priorities for future research. PLANTA 2019, 250, (5).

30. Thomma, B.P.H.J.; Nuernberger, T.; Joosten, M.H.A.J., Of PAMPs and Effectors: The Blurred PTI-ETI Dichotomy. PLANT CELL 2011, 23, (1), 4-15.

31. Wang, R.; Lu, L.; Pan, X.; Hu, Z.; Ling, F.; Yan, Y.; Liu, Y.; Lin, Y., Functional analysis of OsPGIP1 in rice sheath blight resistance. PLANT MOL BIOL 2015, 87, (1-2), 181-191.

32. Xue, X.; Cao, Z.X.; Zhang, X.T.; Wang, Y.; Zhang, Y.F.; Chen, Z.X.; Pan, X.B.; Zuo, S.M., Overexpression of OsOSM1 Enhances Resistance to Rice Sheath Blight. PLANT DIS 2016, 100, (8), 1634-1642.

33. Yang, D.L.; Yang, Y.; He, Z., Roles of plant hormones and their interplay in rice immunity. MOL PLANT 2013, 6, (3), 675-85.

34. Yuan, W.; Jiang, T.; Du K; Chen, H.; Cao, Y.; Xie, J.; Li, M.; Carr, J.P.; Wu, B.; Fan, Z.; Zhou, T., Maize phenylalanine ammonia-lyases contribute to resistance to Sugarcane mosaic virus infection, most likely through positive regulation of salicylic acid accumulation. MOL PLANT PATHOL 2019, 20, (10), 1365-1378.

35. Yu, O.; Jez, J.M., Nature's assembly line: biosynthesis of simple phenylpropanoids and polyketides. The Plant journal : for cell and molecular biology 2008, 54, (4), 750-62.

36. Zakiah, R.M.; Md, A.A.; Md, S.A.; Md, A.R.; Md, R.B.; Md, S.M.; Khandakar, M.I.; Md, A.L.; Mohammad, A.I.K., Morphological and Genetical Variability among Rhizoctonia solani Isolates Causing Sheath Blight Disease of Rice. RICE SCI 2016, 23, (01), 42-50.

37. Zhang, J.; Chen, L.; Fu, C.; Wang, L.; Liu, H.; Cheng, Y.; Li, S.; Deng, Q.; Wang, S.; Zhu, J.; Liang, Y.; Li, P.; Zheng, A., Comparative Transcriptome Analyses of Gene Expression Changes Triggered by Rhizoctonia solani AG1 IA Infection in Resistant and Susceptible Rice Varieties. FRONT PLANT SCI 2017, 8.

38. Zheng, A.; Lin, R.; Zhang, D.; Qin, P.; Xu, L.; Ai, P.; Ding, L.; Wang, Y.; Chen, Y.; Liu, Y.; Sun, Z.; Feng, H.; Liang, X.; Fu, R.; Tang, C.; Li, Q.; Zhang, J.; Xie, Z.; Deng, Q.; Li, S.; Wang, S.; Zhu, J.; Wang, L.; Liu, H.; Li, P., The evolution and pathogenic mechanisms of the rice sheath blight pathogen. NAT COMMUN $2013,4$.

39. Zuo, S.M.; Zhu, Y.J.; Yin, Y.J.; Wang, H.; Zhang, Y.F.; Chen, Z.X.; Gu, S.L.; Pan, X.B., Comparison and Confirmation of Quantitative Trait Loci Conferring Partial Resistance to Rice Sheath Blight on Chromosome 9. PLANT DIS 2014, 98, (7), 957-964.

40. Zuo, S.; Yin, Y.; Zhang, L.; Zhang, Y.; Chen, Z.; Gu, S.; Zhu, L.; Pan, X., Effect and breeding potential of qSB-11(LE), a sheath blight resistance quantitative trait loci from a susceptible rice cultivar. CAN J PLANT SCI 2011, 91, (1), 191-198.

\section{Figures}




\section{Figure 1}

Venn diagram of differentially expressed genes found in both rice cultivars at the same time points after AG1 IA infection. GG 24 h, GG 48, GG72 and GG96 denote the differentially expressed gene sets obtained from Gangyuan8 leaves at 24, 48, 72 and $96 \mathrm{~h}$ after AG1 IA infection, respectively, and YY24 h, YY48 h, YY72 $\mathrm{h}$ and $\mathrm{YY96} \mathrm{h}$ denote the differentially expressed gene sets obtained from Yanfeng47 leaves at 24, 48, 72 and $96 \mathrm{~h}$ after AG1 IA infection.

\section{Figure 2}

Venn diagram of differentially expressed genes in both rice cultivars at different time points after AG1 IA inoculation. GG24 h, GG48 h, GG72 h, and GG96 h denote the differentially expressed gene sets obtained by comparing Gangyuan8 samples at 24, 48, 72 and $96 \mathrm{~h}$ after AG1 IA inoculation, respectively, with the control sample at the same time point, and $Y Y 24 \mathrm{~h}, \mathrm{YY} 48 \mathrm{~h}, \mathrm{YY} 72 \mathrm{~h}$ and $\mathrm{YY} 96 \mathrm{~h}$ denote the differentially expressed gene sets obtained by comparing the GG samples at 24, 48, 72 and $96 \mathrm{~h}$ after AG1 IA inoculation, respectively, with the control sample at the same time point. DEGs, differentially expressed genes; upregulated, upregulated DEGs; downregulated, downregulated DEGs.

\section{Figure 3}

Temporal expression profiles of the 11 clusters of DEGs in the GG and YY leaves.

\section{Figure 4}

Detailed expression profiles of genes related to phenylalanine metabolism.

\section{Figure 5}

Detailed expression profiles of genes related to alpha-linolenic acid metabolism.

\section{Figure 6}

Detailed expression profiles of genes related to plant hormone signal transduction.

\section{Supplementary Files}

This is a list of supplementary files associated with this preprint. Click to download. 
- FigureS1.png

- Figures2.pdf

- FigureS3.pdf

- FigureS4.pdf

- FigureS5.pdf

- FigureS6.pdf

- FigureS7.pdf

- FigureS8.pdf

- TableS1.docx 